\section{Qualidade do processo da assistência pré-natal: unidades básicas de saúde e unidades de Estratégia Saúde da Família em município no Sul do Brasil}

\author{
Quality of prenatal care: traditional primary care \\ and Family Health Strategy units in a city in \\ southern Brazil
}

\author{
1 Programa de Pós-graduação \\ em Epidemiologia \\ Universidade Federal do Rio \\ Grande do Sul, Porto Alegre, \\ Brasil. \\ 2 Departamento de Saúde \\ Coletiva, Universidade \\ Federal de Ciências da Saúde \\ de Porto Alegre, Porto Alegre, \\ Brasil. \\ ${ }^{3}$ Departamento de \\ Estatística, Universidade \\ Federal do Rio Grande do Sul, \\ Porto Alegre, Brasil. \\ Correspondência \\ E. T. R. Anversa \\ Programa de Pós-graduação \\ em Epidemiologia \\ Universidade Federal do Rio \\ Grande do Sul. \\ Av. Nossa Senhora Medianeira \\ 355, Santa Maria, RS \\ 97060-001, Brasil. \\ eleanversa@hotmail.com
}

\begin{abstract}
Prenatal care in traditional primary care units (UBS) and Family Health Strategy units (ESF) was evaluated by a cross-sectional study from July 2009 to February 2010 in Santa Maria, Rio Grande do Sul State, Brazil. Seven hundred and ninety-five postpartum women who had received prenatal care in either of the two types of units were interviewed. Four quality levels were used: level 1 (Kessner index modified by Takeda); level 2, which adds clinical obstetric procedures to level 1; level 3, which adds laboratory tests to level 1; and level 4, which includes all the above parameters. Prenatal care in the Family Health Strategy was superior to that of traditional primary care at all levels, with statistically significant differences in levels 1 and 2. Pregnant women received more guidance and prenatal care was superior in the Family Health Strategy. The study favored the Family Health Strategy, but improvement is still needed in the performance of procedures and laboratory tests in order to enhance prenatal care and strengthen primary care.
\end{abstract}

Prenatal Care; Quality of Health Care; Family Health; Primary Health Care
Elenir Terezinha Rizzetti Anversa 1

Gisele Alsina Nader Bastos 1,2

Luciana Neves Nunes 3

Tatiane da Silva Dal Pizzol 1

\section{Introdução}

A avaliação dos sistemas de saúde constitui-se em uma potente ferramenta norteadora, para gestores e profissionais de saúde. O enfoque mais tradicional de avaliação, proposto por Donabedian 1, está baseado na teoria dos sistemas, que considera os elementos de estrutura, processo e resultados. A avaliação do processo refere-se às ações desenvolvidas nos serviços de saúde, orientada essencialmente para a análise da atuação profissional no cuidado do binômio saúde/ doença da população. Avaliar o processo requer critérios que são delimitados, geralmente, pela resolutividade das ações desenvolvidas.

A qualidade do pré-natal tem sido avaliada por meio do número de consultas e da idade gestacional de ingresso no serviço de saúde. No entanto, estudos demonstram a necessidade de avaliar não apenas o número de consultas, mas também o seu conteúdo ${ }^{2,3}$. Alguns autores questionam o número proposto de consultas para gestantes de baixo risco 4,5,6. Pesquisa realizada em 75 países da América Latina, Ásia e África, demonstrou não haver diferenças significativas em relação ao resultado gestacional entre mulheres com uma mediana de cinco consultas de prénatal quando comparadas àquelas com mediana de oito consultas 5 .

Estudos realizados no Brasil revelaram que mulheres com menor renda familiar, menor escolaridade e não brancas são as que ingressam 
tardiamente no pré-natal e, quando o realizam, este é de mais baixa qualidade, revelando iniquidades sociais presentes na assistência 7,8,9,10.

A avaliação do pré-natal pode contribuir para melhorar a assistência às gestantes, diminuindo os índices de morbimortalidade materna e perinatal 7,11,12. Vários índices são sugeridos pela literatura para avaliar a qualidade, entre os quais destacam-se o índice de Kessner 13, proposto em 1973, e o Adequacy of Prenatal Care Utilization (APNCU), proposto por Kotelchuck 14 em 1994. Takeda 15, em 1993, modificou o índice de Kessner, reduzindo o número de consultas e aumentando a idade gestacional para antes de vinte semanas do início do pré-natal.

Em 2000, o Ministério da Saúde instituiu o Programa de Humanização no Pré-Natal e Nascimento (PHPN) 16; até então, não havia um modelo que normatizasse a assistência às gestantes no Brasil. Esse programa estabeleceu não apenas o número de consultas e a idade gestacional de ingresso, mas elencou, também, exames laboratoriais e ações de educação em saúde, e trouxe a discussão das práticas em saúde e suas bases conceituais, em conformidade com os modelos empregados em todo o mundo 17.

Em 2006, o Brasil adotou a Estratégia Saúde da Família (ESF) como modelo assistencial para reorganizar a atenção primária à saúde ${ }^{18}$. Países com uma potente orientação para a atenção primária à saúde apresentam melhores condições de saúde, custos mais baixos e maior satisfação das pessoas com os sistemas de saúde 19 .

A expansão da ESF e a implementação do PHPN estão entre as estratégias empregadas para a redução da mortalidade materna no Brasil, de modo a atingir os objetivos de desenvolvimento do milênio 20 .

Embora existam vários estudos sobre a adequação do pré-natal, poucos compararam a assistência prestada pelas unidades básicas de saúde (UBS) tradicionais e ESF 21.

O objetivo deste trabalho foi avaliar o processo da atenção pré-natal, verificando se existe diferença na qualidade do pré-natal ofertado nas UBS tradicionais e unidades de ESF de um município no Sul do Brasil.

\section{Metodologia}

O presente trabalho foi realizado no Município de Santa Maria, localizado na região central do Estado do Rio Grande do Sul. Santa Maria possui uma população de 268.965 habitantes, sendo 85.359 o número de mulheres em idade fértil (Departamento de Informática do SUS. http://ta bnet.datasus.gov.br/cgi/deftohtm.exe?ibge/cnv/ poprs, acessado em 10/Mai/2010). O sistema de saúde é composto por 31 unidades, das quais 18 são UBS tradicionais e 13 unidades com ESF, sendo que três unidades da ESF contam com equipe dupla, totalizando 16 equipes. A rede hospitalar tem sete hospitais, cinco privados e dois públicos - Hospital Universitário de Santa Maria (HUSM) e Hospital Municipal Casa de Saúde.

No ano de 2009, 69\% dos partos aconteceram nos dois únicos estabelecimentos de saúde do município que atendem pelo Sistema Único de Saúde (SUS), 44\% ocorreram no HUSM e 25\% no Hospital Municipal Casa de Saúde (Ministério da Saúde. Sistema de Informações de Nascidos Vivos. http:// www.saude.rs.gov.br/ dados/1294405035581Publ_SINASC_2009.xls, acessado em 06/Abr/2011).

O delineamento do estudo foi transversal. Para a estimativa de tamanho da amostra foi considerada uma proporção esperada de qualidade de pré-natal não adequada de $63 \%$ nas UBS tradicionais, de acordo com estudo anterior 22. Com esse parâmetro, e considerando um nível de 95\% de confiança, poder de $90 \%$, razão de não expostos: expostos de 2:1 e uma razão de chances de 2,00 , a amostra estimada foi de 513 pessoas.

Uma equipe de entrevistadores dos cursos de enfermagem da Universidade Federal de Santa Maria (UFSM), Centro Universitário Franciscano (UNIFRA) e Faculdade Integrada de Santa Maria (FISMA), e dois supervisores de campo, foram capacitados para a coleta dos dados. O estudo piloto foi realizado no Hospital Municipal Casa de Saúde para adequação dos instrumentos e procedimentos de coleta de dados.

A coleta de dados ocorreu em três momentos: aplicação da entrevista à puérpera por meio de um questionário padronizado e pré-codificado, seguida pela coleta dos dados no cartão da gestante e, por último, no prontuário. Diariamente, entrevistadores visitavam a maternidade do Hospital Municipal Casa de Saúde e o Centro Obstétrico e Maternidade do HUSM, para realizar a coleta de dados. As entrevistas ocorreram respeitando-se o prazo de quatro a 48 horas após o parto, e somente foram realizadas após a apresentação e assinatura do Termo de Consentimento Livre e Esclarecido.

Foram incluídas as puérperas que realizaram pré-natal nas unidades de saúde de Santa Maria, no período de julho de 2009 a fevereiro de 2010. Excluiu-se: as puérperas que iniciaram o pré-natal nas unidades de saúde, mas foram referidas para o ambulatório de alto risco; as que realizaram seu pré-natal na rede privada; as que não possuíam o cartão da gestante no momento da entrevista; e as puérperas que não realizaram nenhuma consulta de pré-natal, pela impossibi- 
lidade de avaliar a qualidade do pré-natal, pois não acessaram os serviços de saúde. Assim, foram entrevistadas, consecutivamente, todas as puérperas internadas nos dois locais de coleta dos dados.

As puérperas foram questionadas sobre características demográficas, nível socioeconômico, história reprodutiva, assistência recebida durante o pré-natal, planejamento da gravidez e realização de ultrassonografia gestacional. Foram perguntadas se receberam, durante o prénatal, orientações sobre aleitamento materno, anticoncepção pós-parto, consulta de puerpério, sinais e sintomas anormais durante o período de gestação, assistência ao recém-nascido, tipo de parto, participação em grupo de gestantes ou orientações em sala de espera e realização do exame anti-HIV. Também foram questionadas sobre a realização do exame clínico de mamas e citopatológico, e se receberam suplementação de ferro e vacina antitetânica.

As informações obtidas no cartão da gestante foram: unidade de saúde onde realizou o pré-natal; data da última menstruação (DUM); registro da idade gestacional na primeira consulta e número de consultas realizadas, com a respectiva idade gestacional; dados sobre a realização de exames laboratoriais; exames de ultrassonografia obstétrica; peso; pressão arterial; altura uterina; ausculta de batimentos cardiofetais; movimentos fetais e toque vaginal. Quando a DUM não constava no cartão da gestante, recorreu-se ao prontuário e, quando não encontrada, utilizouse a idade gestacional do parto e realizou-se a regressão por meio de disco gestacional.

Foram coletados os seguintes dados do prontuário: data de internação, horário de internação, idade gestacional do parto.

Para o desfecho principal do estudo, qualidade do pré-natal, foram estabelecidos quatro níveis de qualidade.

Nível 1: qualidade do pré-natal, avaliada pelo número de consultas e início do pré-natal, de acordo com o índice de Kessner modificado por Takeda 15 . Classifica a qualidade em três categorias: adequada (quando no cartão da gestante estavam registradas seis ou mais consultas e o início do pré-natal antes de 20 semanas); inadequada (quando no cartão da gestante estava registrado o início do pré-natal após 28 semanas, ou menos de três consultas); intermediária (nas demais situações).

Nível 2: qualidade do pré-natal avaliada pelo número de consultas, início do pré-natal e procedimentos conforme manual técnico do Ministério da Saúde 23 (nível 1+ nível 2). Para a análise dos procedimentos realizados durante a assistência pré-natal utilizou-se o manual técnico do Ministério da Saúde 23 , tendo em vista que o PHPN 16 não estabelece quais os procedimentos que devem ser realizados durante o pré-natal. Categorias: adequada (quando no cartão da gestante estavam registradas seis ou mais consultas, início do pré-natal antes de 20 semanas de gestação, cinco ou mais registros de cada um dos seguintes procedimentos: pressão arterial, peso, idade gestacional, registros da altura uterina, e quatro ou mais registros dos batimentos cardiofetais e movimentos fetais); inadequada (quando no cartão da gestante estava registrado o início do pré-natal após 28 semanas de gestação, ou menos de três consultas, dois ou menos registros da altura uterina, idade gestacional, pressão arterial, peso, batimentos cardiofetais e movimentos fetais); intermediária (nas demais situações).

Nível 3: qualidade do pré-natal avaliada pelo número de consultas, início do pré-natal e exames laboratoriais preconizados pelo PHPN 17 (nível 1+ nível 3). Optou-se pelos exames próximos a trinta semanas (28-32), conforme preconizado pelo manual técnico do Ministério da Saúde 23 , pois o PHPN 16 recomenda a realização dos exames na primeira consulta e na 30a semana de gestação. Categorias: adequada [quando no cartão da gestante estavam registradas seis ou mais consultas, início do pré-natal antes de vinte semanas de gestação e um registro dos exames: tipagem sanguínea e fator $\mathrm{RH}(\mathrm{ABO} / \mathrm{RH})$, hemoglobina e hematócrito $(\mathrm{Hb} / \mathrm{Ht})$, exame de sífilis (VDRL), exame qualitativo de urina (EQU), glicemia de jejum, teste anti-HIV e toxoplasmose na primeira consulta, e nova realização dos exames próximo à 30a semana de gestação: VDRL, EQU, glicemia de jejum, teste anti-HIV, além de um exame HBsAg (antígeno de superfície do vírus da hepatite B)]; inadequada (quando no cartão da gestante estava registrado o início do pré-natal após 28 semanas ou menos de três consultas e nenhum registro dos exames); intermediária (em todas as demais situações).

Nível 4: qualidade do pré-natal avaliada pelo número de consultas e início do pré-natal, procedimentos realizados durante o pré-natal e exames laboratoriais (nível $1+$ nível $2+$ nível 3). Categorias: adequada (quando no cartão da gestante estavam registradas seis ou mais consultas de pré-natal e início do pré-natal antes de vinte semanas, cinco ou mais registros dos procedimentos de aferição da pressão arterial, peso, idade gestacional, altura uterina e quatro ou mais registros dos batimentos cardiofetais e movimentos fetais e pelo menos um dos exames: $\mathrm{ABO} / \mathrm{RH}, \mathrm{Ht} / \mathrm{Hb}, \mathrm{VDRL}, \mathrm{EQU}$, glicemia de jejum, teste anti-HIV e toxoplasmose na primeira consulta, e nova realização dos exames próximo à 30a semana de gestação: VDRL, EQU, glicemia de 
jejum, teste anti-HIV e um exame HBsAg); inadequada (quando no cartão da gestante estava registrado o início do pré-natal após 28 semanas, ou menos de três consultas, dois ou menos registros da altura uterina, idade gestacional, pressão arterial, peso, batimentos cardiofetais, movimentos fetais e nenhum registro dos exames; intermediária (nas demais situações).

A variável preditora foi o local de realização do pré-natal (UBS tradicional ou ESF), investigando se havia diferença da qualidade entre os dois modelos de atenção.

Os dados foram armazenados no programa Epi Info, versão 3.5.1 (Centres for Disease Control and Prevention, Atlanta, Estados Unidos), com dupla digitação para detectar possíveis erros durante a mesma. As análises estatísticas foram realizadas no programa SPSS, versão 18.0 (SPSS Inc., Chicago, Estados Unidos). A análise dos dados envolveu procedimentos de estatística descritiva e análises bivariadas. Utilizou-se o teste do qui-quadrado para comparação das proporções.

O projeto de pesquisa foi aprovado pelo Comitê de Ética do Centro Universitário Franciscano (UNIFRA) (registro CEP/UNIFRA: 090.2009.2 e registro no CONEP no. 1246) e pelo Comitê de Ética do Hospital Nossa Senhora da Conceição (GHC SISNEP nº. 272956, protocolo 09-091).

\section{Resultados}

O total de puérperas abordadas para a entrevista foi de 910 , sendo que destas, 21 (2\%) foram perdas/recusas e 13 (1\%) adolescentes sem responsável que pudesse assinar o Termo de Consentimento Livre e Esclarecido no momento da entrevista. Outros motivos de exclusões foram: surto psicótico $1(0 \%)$, isolamento devido à gripe por H1N1 2 (0\%) e óbito materno por H1N1 $1(0 \%)$. Não realizaram o pré-natal 38 puérperas (4\%) e 39 (4\%) não possuíam o cartão no momento da entrevista (por esquecimento em casa, não ter sido encontrado na unidade de internação ou por já ter sido enviado para casa). Participaram do estudo 795 puérperas, das quais 501 (63\%) foram atendidas no HUSM e 294 (37\%) no Hospital Municipal Casa de Saúde.

Das 795 puérperas, $68 \%$ realizaram o pré-natal nas UBS tradicionais e $32 \%$ na ESF. As principais características das puérperas, de acordo com o local de realização do pré-natal, são apresentadas na Tabela 1. As gestantes atendidas nas UBS tradicionais não diferiram significativamente das atendidas na ESF em relação às características demográficas, socioeconômicas e reprodutivas investigadas.
As puérperas apresentaram idade média de 25 anos (DP $=6,8$ ), variando de 13 a 48 anos. Aquelas que frequentaram as UBS tradicionais tinham idade média de 25,2 anos ( $\mathrm{DP}=6,9$ ) e as que frequentaram as unidades da ESF tinham idade média de 24,4 (DP =6,4). As que consultaram nas UBS tradicionais apresentaram média de anos completos de estudos de 8,4 (DP = $2,4)$, maior do que as usuárias da ESF que foi 8,0 anos $(\mathrm{DP}=2,4)$. Em relação à renda familiar observa-se que as puérperas que frequentaram as UBS tinham uma renda média de 3,18 salários mínimos ( $\mathrm{DP}=0,99$ ). Para as puérperas da ESF a renda média foi de 3,01 salários mínimos $(\mathrm{DP}=1,05)$.

Das gestações planejadas, $41 \%$ foram de usuárias das UBS tradicionais e 43\% da ESF. O número médio de consultas nas UBS tradicionais foi de 6,3 (DP = 2,6) e a idade gestacional média no início do pré-natal foi de 16 semanas ( $\mathrm{DP}=7,6$ ). Nas unidades da ESF as médias foram 6,8 (DP = 2,6) e 14 semanas ( $\mathrm{DP}=6,3)$, respectivamente.

A maioria dos procedimentos preconizados pelo manual técnico do Ministério da Saúde 23 foi realizada com maior frequência nas gestantes atendidas pela ESF (Tabela 2). Os procedimentos realizados com maior frequência nas UBS tradicionais foram o toque vaginal e a ultrassonografia. A realização de ultrassonografia não foi avaliada como parâmetro de qualidade do pré-natal.

Na Tabela 3, são apresentados os exames laboratoriais realizados por local de atendimento. A realização dos exames que devem ser solicitados na primeira consulta, conforme preconizado pelo PHPN, ocorreu em menos de $40 \%$ dos casos. Os exames realizados próximos à 30a semana de gestação foram mais frequentes quando comparados com os solicitados na primeira consulta. Não foram verificadas diferenças na solicitação de exames na primeira consulta e na 30 a semana nos dois modelos de atenção.

As gestantes que realizaram o pré-natal na ESF receberam mais orientações durante o período que as atendidas em UBS tradicionais (Tabela 4 ).

A Tabela 5 apresenta a adequação do prénatal nos quatro níveis de qualidade propostos, para todas as gestantes e por modelo de atenção. A assistência pré-natal foi classificada como adequada para 59\% das gestantes, de acordo com o nível 1 . Os percentuais de adequação do pré-natal na ESF foram superiores aos encontrados nas UBS tradicionais em todos os níveis propostos, com diferenças estatisticamente significativas para o nível $1(\mathrm{p}=0,02)$ e o nível $2(\mathrm{p}=0,01)$. 
Distribuição da amostra de acordo com as características demográficas, socioeconômicas e reprodutivas, conforme local de realização do pré-natal. Município de Santa Maria, Rio Grande do Sul, Brasil ( $=795)$.

\begin{tabular}{|c|c|c|c|c|c|c|c|}
\hline \multirow[t]{2}{*}{ Variável } & \multicolumn{2}{|c|}{ Todas } & \multicolumn{2}{|c|}{ UBS } & \multicolumn{2}{|c|}{ ESF } & \multirow[t]{2}{*}{ Valor de $p$} \\
\hline & $\mathbf{n}$ & $\%$ & $\mathbf{n}$ & $\%$ & $\mathbf{n}$ & $\%$ & \\
\hline \multicolumn{8}{|l|}{ Faixa etária (anos) } \\
\hline $13-19$ & 194 & 24 & 129 & 24 & 65 & 26 & 0,08 \\
\hline $20-34$ & 507 & 64 & 343 & 63 & 164 & 65 & 0,09 \\
\hline 35 ou mais & 94 & 12 & 72 & 13 & 22 & 9 & \\
\hline \multicolumn{8}{|l|}{ Escolaridade (anos) } \\
\hline $0-8$ & 452 & 57 & 297 & 55 & 155 & 62 & 0,28 \\
\hline $9-11$ & 313 & 39 & 225 & 41 & 88 & 35 & 0,87 \\
\hline 12 ou mais & 30 & 4 & 22 & 4 & 8 & 3 & \\
\hline \multicolumn{8}{|l|}{ Raça autorreferida } \\
\hline Branca & 469 & 59 & 324 & 60 & 145 & 58 & 0,57 \\
\hline Não branca & 323 & 41 & 217 & 40 & 106 & 42 & \\
\hline \multicolumn{8}{|l|}{ Situação conjugal } \\
\hline Com companheiro & 686 & 86 & 467 & 86 & 219 & 87 & 0,59 \\
\hline Solteira, sem companheiro ou separada & 109 & 14 & 77 & 14 & 32 & 13 & \\
\hline \multicolumn{8}{|l|}{ Renda familiar (salários mínimos) * } \\
\hline Até 1 & 187 & 24 & 118 & 22 & 69 & 28 & 0,09 \\
\hline $1-3$ & 522 & 66 & 363 & 67 & 159 & 64 & 0,43 \\
\hline Acima de 3 & 84 & 11 & 62 & 12 & 22 & 9 & \\
\hline \multicolumn{8}{|l|}{ História reprodutiva prévia } \\
\hline Primigesta & 322 & 41 & 230 & 42 & 92 & 37 & 0,82 \\
\hline 2-3 gestações & 355 & 45 & 231 & 43 & 124 & 49 & 0,30 \\
\hline Mais de 3 gestações & 118 & 15 & 83 & 15 & 35 & 14 & \\
\hline \multicolumn{8}{|l|}{ Planejamento gravidez atual } \\
\hline $\operatorname{Sim}$ & 333 & 42 & 225 & 41 & 108 & 43 & 0,66 \\
\hline Não & 462 & 58 & 319 & 59 & 143 & 57 & \\
\hline Total & 795 & 100 & 544 & 100 & 251 & 100 & \\
\hline
\end{tabular}

ESF: Estratégia Saúde da Família; UBS: unidades básicas de saúde;

* Salário mínimo: R\$ 460,00.

\section{Discussão}

No presente estudo analisamos a qualidade do processo da assistência pré-natal no Município de Santa Maria, identificando diferenças na assistência pré-natal realizada nas UBS tradicionais e nas ESF, considerando número de consultas, início do pré-natal, procedimentos, exames realizados e orientações recebidas. Com base nos níveis de qualidade propostos, constatamos que a assistência prestada foi insatisfatória nos dois modelos de atenção, sendo que a qualidade da assistência pré-natal na ESF foi superior à das UBS tradicionais.

Com base em uma amostra representativa da população sob investigação, com baixo percentual de perdas ou recusas, comparamos, de forma inédita, a assistência pré-natal nos dois modelos de atenção primária à saúde, utilizando como critérios de avaliação quatro níveis de qualidade. As comparações realizadas neste trabalho foram baseadas em publicações anteriores sobre a qualidade do pré-natal que não avaliaram especificamente a assistência pré-natal na ESF.

A média de consultas de pré-natal realizadas pelas gestantes da ESF $(6,8)$ é superior à encontrada em estudos anteriores (5,3 a 6,5) 2,9,22,24, mas inferior à verificada por outros autores $(7,6 \mathrm{a}$ $7,4) 3,7$. O ingresso precoce no pré-natal das gestantes da ESF foi superior ao de gestantes paulistas 2 , com média de 20,7 semanas, e mineiras ${ }^{24}$, com 17,4 semanas, e inferior às gestantes de Rio Grande (Rio Grande do Sul) 7, com ingresso na 13a semana de gestação. 
Número de gestantes com procedimentos registrados conforme local de realização do pré-natal. Município de Santa Maria, Rio Grande do Sul, Brasil $(n=795)$.

\begin{tabular}{|c|c|c|c|c|c|c|c|}
\hline \multirow[t]{3}{*}{ Variável } & \multicolumn{7}{|c|}{ Número de procedimentos registrados no cartão da gestante } \\
\hline & \multicolumn{2}{|c|}{ Todas } & \multicolumn{2}{|c|}{ UBS } & \multicolumn{2}{|c|}{ ESF } & \multirow[t]{2}{*}{ Valor de $p$} \\
\hline & $\mathbf{n}$ & $\%$ & n & $\%$ & $\mathrm{n}$ & $\%$ & \\
\hline \multicolumn{8}{|c|}{ Idade gestacional } \\
\hline 2 ou menos & 108 & 14 & 84 & 15 & 24 & 10 & $<0,001$ \\
\hline $3-4$ & 154 & 19 & 12 & 23 & 32 & 13 & \\
\hline 5 ou mais & 533 & 67 & 338 & 62 & 195 & 78 & \\
\hline \multicolumn{8}{|l|}{ Peso } \\
\hline 2 ou menos & 64 & 8 & 49 & 9 & 15 & 6 & 0,02 \\
\hline $3-4$ & 163 & 21 & 123 & 23 & 40 & 16 & \\
\hline 5 ou mais & 568 & 71 & 372 & 68 & 196 & 78 & \\
\hline \multicolumn{8}{|l|}{ Pressão arterial } \\
\hline 2 ou menos & 58 & 7 & 44 & 8 & 14 & 6 & 0,02 \\
\hline $3-4$ & 157 & 20 & 119 & 22 & 38 & 15 & \\
\hline 5 ou mais & 580 & 73 & 381 & 70 & 199 & 79 & \\
\hline \multicolumn{8}{|l|}{ Altura uterina } \\
\hline 2 ou menos & 96 & 12 & 68 & 13 & 28 & 11 & 0,02 \\
\hline $3-4$ & 206 & 26 & 156 & 29 & 50 & 20 & \\
\hline 5 ou mais & 493 & 62 & 320 & 59 & 173 & 69 & \\
\hline \multicolumn{8}{|c|}{ Batimentos cardiofetais } \\
\hline 2 ou menos & 105 & 13 & 77 & 14 & 28 & 11 & 0,13 \\
\hline 3 & 99 & 13 & 74 & 14 & 25 & 10 & \\
\hline 4 ou mais & 591 & 74 & 393 & 72 & 198 & 79 & \\
\hline \multicolumn{8}{|c|}{ Movimentos fetais } \\
\hline 2 ou menos & 121 & 15 & 92 & 17 & 29 & 12 & 0,01 \\
\hline 3 & 102 & 13 & 79 & 15 & 23 & 9 & \\
\hline 4 ou mais & 572 & 72 & 373 & 69 & 199 & 79 & \\
\hline \multicolumn{8}{|l|}{ Toque vaginal } \\
\hline 2 ou menos & 627 & 79 & 405 & 75 & 222 & 88 & $<0,001$ \\
\hline $3-4$ & 109 & 14 & 92 & 17 & 17 & 7 & \\
\hline \multirow[t]{4}{*}{5 ou mais } & 59 & 7 & 47 & 9 & 12 & 5 & \\
\hline & \multicolumn{7}{|c|}{ Realização de procedimentos referidos pelas gestantes } \\
\hline & \multicolumn{2}{|c|}{ Todas } & \multicolumn{2}{|c|}{ UBS } & \multicolumn{2}{|c|}{ ESF } & Valor de $\mathrm{p}$ \\
\hline & n & $\%$ & $\mathrm{n}$ & $\%$ & $\mathrm{n}$ & $\%$ & \\
\hline \multicolumn{8}{|c|}{ Clínico das mamas } \\
\hline Não & 371 & 47 & 281 & 52 & 90 & 36 & $<0,001$ \\
\hline Sim & 421 & 53 & 261 & 48 & 160 & 64 & \\
\hline \multicolumn{8}{|l|}{ Utrassonografia } \\
\hline Nenhuma & 49 & 6 & 31 & 6 & 18 & 7 & $<0,001$ \\
\hline 1 & 213 & 27 & 108 & 20 & 105 & 42 & \\
\hline 2 ou mais & 533 & 67 & 405 & 74 & 128 & 51 & \\
\hline Suplementação & & & & & & & \\
\hline Não & 111 & 14 & 87 & 16 & 24 & 10 & 0,01 \\
\hline Sim & 679 & 86 & 433 & 84 & 226 & 90 & \\
\hline Citopatológico & & & & & & & \\
\hline Não & 508 & 65 & 376 & 71 & 132 & 53 & $<0,001$ \\
\hline Sim & 271 & 35 & 153 & 29 & 118 & 47 & \\
\hline Vacina antitetân & & & & & & & \\
\hline Não & 287 & 36 & 210 & 39 & 77 & 31 & 0,03 \\
\hline Sim & 508 & 64 & 334 & 61 & 174 & 69 & \\
\hline
\end{tabular}

ESF: Estratégia Saúde da Família; UBS: unidades básicas de saúde. 
Exames laboratoriais conforme local de realização do pré-natal. Município de Santa Maria, Rio Grande do Sul, Brasil (n = 795).

\begin{tabular}{|c|c|c|c|c|c|c|c|}
\hline \multirow[t]{3}{*}{ Variável } & \multicolumn{7}{|c|}{ Exames realizados na primeira consulta } \\
\hline & \multicolumn{2}{|c|}{ Todas } & \multicolumn{2}{|c|}{ UBS } & \multicolumn{2}{|c|}{ ESF } & \multirow{2}{*}{$\begin{array}{l}\text { Valor } \\
\text { de } p\end{array}$} \\
\hline & n & $\%$ & n & $\%$ & $\mathrm{n}$ & $\%$ & \\
\hline \multicolumn{8}{|l|}{$\mathrm{ABO} / \mathrm{RH}$} \\
\hline Não & 488 & 61 & 335 & 62 & 153 & 61 & 0,87 \\
\hline Sim & 307 & 39 & 209 & 38 & 98 & 39 & \\
\hline \multicolumn{8}{|l|}{$\mathrm{Hb} / \mathrm{Ht}$} \\
\hline Não & 492 & 62 & 338 & 62 & 154 & 61 & 0,83 \\
\hline Sim & 303 & 38 & 206 & 38 & 97 & 39 & \\
\hline \multicolumn{8}{|l|}{ EQU } \\
\hline Não & 511 & 64 & 350 & 64 & 161 & 64 & 0,96 \\
\hline Sim & 284 & 36 & 194 & 36 & 90 & 36 & \\
\hline \multicolumn{8}{|c|}{ Glicemia de jejum } \\
\hline Não & 504 & 63 & 347 & 64 & 157 & 63 & 0,75 \\
\hline Sim & 291 & 37 & 197 & 36 & 94 & 38 & \\
\hline \multicolumn{8}{|l|}{ VDRL } \\
\hline Não & 513 & 65 & 353 & 65 & 160 & 64 & 0,75 \\
\hline Sim & 282 & 36 & 191 & 35 & 91 & 36 & \\
\hline \multicolumn{8}{|l|}{ Anti-HIV } \\
\hline Não & 518 & 65 & 356 & 65 & 162 & 65 & 0,80 \\
\hline Sim & 277 & 35 & 188 & 35 & 89 & 36 & \\
\hline \multicolumn{8}{|c|}{ Toxoplasmose } \\
\hline Não & 538 & 68 & 371 & 68 & 167 & 67 & 0,64 \\
\hline \multirow[t]{4}{*}{ Sim } & 257 & 32 & 173 & 32 & 84 & 34 & \\
\hline & \multicolumn{7}{|c|}{ Exames realizados com 30 semanas } \\
\hline & \multicolumn{2}{|c|}{ Todas } & \multicolumn{2}{|c|}{ UBS } & \multicolumn{2}{|c|}{ ESF } & Valor \\
\hline & $\mathbf{n}$ & $\%$ & $\mathbf{n}$ & $\%$ & $\mathrm{n}$ & $\%$ & de $p$ \\
\hline \multicolumn{8}{|l|}{ EQU } \\
\hline Não & 409 & 51 & 284 & 52 & 125 & 50 & 0,53 \\
\hline Sim & 386 & 49 & 260 & 48 & 126 & 50 & \\
\hline \multicolumn{8}{|c|}{ Glicemia de jejum } \\
\hline Não & 401 & 50 & 274 & 50 & 127 & 51 & 0,95 \\
\hline Sim & 394 & 50 & 270 & 50 & 124 & 49 & \\
\hline \multicolumn{8}{|l|}{ VDRL } \\
\hline Não & 497 & 63 & 349 & 64 & 148 & 59 & 0,16 \\
\hline Sim & 298 & 38 & 195 & 36 & 103 & 41 & \\
\hline \multicolumn{8}{|l|}{ HIV } \\
\hline Não & 503 & 63 & 346 & 64 & 157 & 63 & 0,77 \\
\hline Sim & 292 & 37 & 198 & 36 & 94 & 38 & \\
\hline \multicolumn{8}{|l|}{$\mathrm{HbSAg}$} \\
\hline Não & 445 & 56 & 305 & 56 & 140 & 56 & 0,94 \\
\hline Sim & 350 & 44 & 239 & 44 & 111 & 44 & \\
\hline
\end{tabular}

ABO/RH: tipagem sanguínea e fator RH; EQU: exame qualitativo de urina; ESF: Estratégia Saúde da Família;

HBsAg: antígeno de superfície do vírus da hepatite $\mathrm{B} ; \mathrm{Hb} / \mathrm{Ht}$ : hemoglobina e hematócrito; UBS: unidades básicas de saúde; VDRL: exame de sífilis. 
Orientações recebidas durante o pré-natal conforme local de realização do pré-natal. Município de Santa Maria, Rio Grande do Sul, Brasil ( $n=795)$.

\begin{tabular}{|c|c|c|c|c|c|c|c|}
\hline \multirow[t]{2}{*}{ Variável } & \multicolumn{2}{|c|}{ Todas } & \multicolumn{2}{|c|}{ UBS } & \multicolumn{2}{|c|}{ ESF } & \multirow[t]{2}{*}{ Valor de $p$} \\
\hline & n & $\%$ & n & $\%$ & $\mathbf{n}$ & $\%$ & \\
\hline \multicolumn{8}{|l|}{ Aleitamento materno } \\
\hline Sim & 509 & 64 & 324 & 60 & 185 & 74 & $<0,001$ \\
\hline Não & 285 & 36 & 220 & 40 & 65 & 26 & \\
\hline \multicolumn{8}{|l|}{ Anticoncepção pós-parto } \\
\hline $\operatorname{Sim}$ & 339 & 43 & 214 & 39 & 125 & 50 & 0,01 \\
\hline Não & 455 & 57 & 339 & 61 & 126 & 50 & \\
\hline Consulta de retorno puerpério & & & & & & & $<0,001$ \\
\hline Sim & 384 & 48 & 235 & 43 & 149 & 59 & \\
\hline Não & 409 & 52 & 307 & 57 & 102 & 41 & \\
\hline \multicolumn{8}{|c|}{ Sinais e sintomas anormais na gestação } \\
\hline Sim & 486 & 62 & 333 & 62 & 153 & 61 & \\
\hline Não & 303 & 38 & 205 & 38 & 98 & 39 & 0,80 \\
\hline \multicolumn{8}{|l|}{ Cuidados com o recém-nascido } \\
\hline Sim & 539 & 68 & 350 & 65 & 189 & 75 & $<0,001$ \\
\hline Não & 255 & 32 & 193 & 36 & 62 & 25 & \\
\hline \multicolumn{8}{|l|}{ Orientações sobre o tipo de parto } \\
\hline Sim & 277 & 35 & 175 & 32 & 102 & 59 & \\
\hline Não & 517 & 65 & 368 & 68 & 149 & 41 & $<0,001$ \\
\hline \multicolumn{8}{|l|}{ Orientações sobre HIV } \\
\hline $\operatorname{Sim}$ & 602 & 76 & 390 & 72 & 212 & 85 & $<0,001$ \\
\hline Não & 192 & 24 & 153 & 28 & 39 & 16 & \\
\hline \multicolumn{8}{|c|}{ Participação em grupos de gestantes } \\
\hline Sim & 136 & 17 & 93 & 17 & 43 & 17 & \\
\hline Não & 658 & 83 & 450 & 83 & 208 & 83 & 0,99 \\
\hline
\end{tabular}

ESF: Estratégia Saúde da Família; UBS: unidades básicas de saúde.

Menos de $20 \%$ das gestantes, nos dois modelos de atenção, participaram de grupo de gestantes ou orientações em sala de espera, percentuais inferiores aos encontrados na literatura $(43 \%$ a $83 \%$ ) 9,21 . O pré-natal é um momento singular e oportuno para desenvolver ações educativas, podendo ser realizadas nas unidades de saúde, por intermédio de grupos de gestantes, na sala de espera, ou individualmente. Essa estratégia de trabalho permite a integração de profissionais e gestantes, constituindo um momento de acolhida, escuta, vínculo, de compartilhamento de experiências, trocas mútuas, fortalecimento de conhecimentos e esclarecimento de dúvidas. Nosso estudo apontou que as orientações menos recebidas pelas gestantes foram sobre a anticoncepção pós-parto, consulta de puerpério e orientações sobre tipo de parto.

Ao ser avaliada a qualidade do pré-natal, de acordo com índice de Kessner modificado por
Takeda 15 (nível 1), o pré-natal foi classificado como adequado para $59 \%$ das gestantes. Para as gestantes das UBS a adequação foi de $56 \%$ e para as da ESF de $65 \%$. Em estudos prévios que utilizaram o índice de Kessner modificado, os percentuais variaram de $26 \%$ a $83 \%$ 2,3,7,15,22,24 . A adequação do pré-natal nas UBS foi inferior à verificada em Pelotas (Rio Grande do Sul), em 19983 (83\%), e em Rio Grande, em 20097 (65\%). A adequação do pré-natal da ESF foi praticamente igual à encontrada no estudo realizado em Rio Grande, em 2009 7, e inferior ao de Pelotas, em 1998 3. Os estudos conduzidos antes de 2009 2,3,15,22,24, com exceção do realizado por Halpern et al. 3 , demonstraram escores de pré-natal bem abaixo do verificado no presente trabalho, achado que pode ser reflexo da não implantação das políticas públicas há oito anos ou mais. Nesse transcurso, houve ampliação dos serviços de saúde, inclusão de novas equipes da 
Adequação do pré-natal nos quatro níveis de qualidade, por local de realização do pré-natal. Município de Santa Maria, Rio Grande do Sul, Brasil ( $\mathrm{n}=795)$.

\begin{tabular}{|c|c|c|c|c|c|c|c|}
\hline \multirow[t]{2}{*}{ Adequação } & \multicolumn{2}{|c|}{ Todas } & \multicolumn{2}{|c|}{ UBS } & \multicolumn{2}{|c|}{ ESF } & \multirow{2}{*}{$\begin{array}{l}\text { Valor } \\
\text { de } p\end{array}$} \\
\hline & n & $\%$ & $\mathbf{n}$ & $\%$ & $\mathbf{n}$ & $\%$ & \\
\hline \multicolumn{8}{|l|}{ Nível 1} \\
\hline Adequado & 467 & 59 & 304 & 56 & 163 & 65 & 0,02 \\
\hline Intermediário & 301 & 38 & 217 & 40 & 84 & 33 & \\
\hline Inadequado & 27 & 3 & 23 & 4 & 4 & 2 & \\
\hline \multicolumn{8}{|l|}{ Nível 2} \\
\hline Adequado & 366 & 46 & 232 & 43 & 134 & 53 & 0,01 \\
\hline Intermediário & 402 & 51 & 289 & 53 & 113 & 45 & \\
\hline Inadequado & 27 & 3 & 23 & 4 & 4 & 2 & \\
\hline \multicolumn{8}{|l|}{ Nível 3} \\
\hline Adequado & 62 & 8 & 38 & 7 & 24 & 10 & 0,45 \\
\hline Intermediário & 723 & 91 & 499 & 92 & 224 & 89 & \\
\hline Inadequado & 10 & 1 & 7 & 1 & 3 & 1 & \\
\hline \multicolumn{8}{|l|}{ Nível 4} \\
\hline Adequado & 58 & 7 & 34 & 6 & 24 & 10 & 0,25 \\
\hline Intermediário & 727 & 91 & 503 & 92 & 224 & 89 & \\
\hline Inadequado & 10 & 1 & 7 & 2 & 3 & 2 & \\
\hline
\end{tabular}

ESF: Estratégia Saúde da Família; UBS: unidades básicas de saúde;

Nível 1: Kessner modificado (número de consultas e início do pré-natal);

Nível 2: nível 1 + procedimentos clinico-obstétricos;

Nível 3: nível $1+$ exames laboratoriais;

Nível 4: nível 1 + procedimentos clinico-obstétricos + exames laboratoriais.

ESF, e desenhada a nova Política Nacional de Atenção Básica.

A assistência pré-natal foi adequada em $46 \%$ dos casos quando adicionamos procedimentos clinico-obstétricos ao nível 1, percentual superior ao encontrado por outros autores, que variaram de $5 \%$ a $38 \% 2,22$. Um dos fatores que contribuiu para escores superiores, neste trabalho, foi a forma de avaliação proposta, que adicionou ao índice de Kessner modificado a avaliação de exames e procedimentos, isoladamente. No estudo realizado em Pelotas, em 2001 22, foi adicionado ao índice de Kessner modificado exames e procedimentos, simultaneamente. Em estudo realizado em São Paulo ${ }^{2}$, foi utilizado como critério o maior número de vezes em que foram verificados os procedimentos a serem realizados durante o pré-natal. Aferição da pressão arterial, ausculta dos batimentos cardiofetais, movimentos fetais e realização de dois ou menos toques vaginais, apresentaram maiores percentuais de registros nos dois modelos de atenção, em contraponto com a medida da altura uterina, procedimento menos realizado nos dois modelos de atenção.
O nível 3, que incluiu exames laboratoriais no nível 1, apresentou redução na adequação do pré-natal em relação ao nível 1 (7\% na UBS e $10 \%$ na ESF). O trabalho que avaliou estrutura e processo do pré-natal em Pelotas, em 2001 22, verificou alteração na adequação do pré-natal quando adicionados exames (de 37\% declinou para $31 \%$ ), ao contrário do estudo realizado em Juiz de Fora (Minas Gerais), em 2003 24, que registrou uma redução na adequação de $28 \%$ para $2 \%$. Em outro estudo 2, quando adicionados exames preconizados pelo PHPN, nenhum pré-natal foi classificado como adequado.

O trabalho demonstrou baixo percentual de gestantes que realizaram os exames preconizados pelo PHPN nos dois modelos de atenção. A realização dos exames laboratoriais durante a gestação é um período oportuno para prevenir, identificar e corrigir as anormalidades que possam afetar a gestante e seu concepto, e instituir tratamento de doenças já existentes ou que possam ocorrer durante a gestação. Em Santa Maria, os exames são coletados nas unidades de saúde, facilitando o acesso das usuárias. Embora 
os exames tenham sido registrados no cartão da gestante, o período de solicitação ou de retorno aos profissionais não atendeu aos períodos preconizados, para a maioria dos casos. Essas informações sugerem que as consultas de prénatal não estão sendo realizadas no período recomendado pelo programa, o que pode evidenciar fragilidades na organização do sistema de saúde do município.

O controle e vigilância sistemáticos sobre prontuários e cartões das gestantes poderia ser implementado para melhorar a qualidade da atenção no que diz respeito a exames e procedimentos. Percebe-se uma desvalorização nos registros, não apenas considerando a importância dos dados para os sistemas de informação, mas, principalmente, na identificação do perfil epidemiológico da população, identificando fragilidades, necessidades incisivas para tomada de decisão como, também, um importante instrumento legal. Ceccim \& Ferla 25, afirmam que o "conhecimento e o acompanhamento do impacto das ações em saúde devem ser viabilizados por meio de indicadores adequados, o que implica na existência de registros locais fáceis, confiáveis e contínuos" (p. 175).

Na avaliação da qualidade do pré-natal nível 4, que considerou todos os critérios dos níveis anteriores, o escore de adequação foi extremamente inferior ao do nível 1, nos dois modelos de atenção. Esses achados são semelhantes aos verificados por outros autores, cujos percentuais variaram de $0 \%$ a $5 \%$ 2,22,24, evidenciando a necessidade de outro olhar para a qualidade do pré-natal, além do início e números de consultas 2,3,26.

No presente trabalho, os percentuais de inadequação foram menores do que em outros estudos 2,3,9,22,24, possivelmente em consequência da exclusão das puérperas que não haviam realizado nenhuma consulta de pré-natal.

A adequação do pré-natal em todos os níveis avaliados foi favorável às gestantes que realizaram o pré-natal na ESF, em comparação às que foram atendidas nas UBS. Essa diferença pode ser atribuída ao processo de trabalho, presença dos agentes comunitários de saúde, busca ativa das gestantes, formação de vínculo com os profissionais, registros nos cartões da gestante, trabalho em equipe e adscrição da clientela, conforme preconizado pela Política Nacional de Atenção Básica 18 . As gestantes atendidas pela ESF receberam mais orientações durante a gestação e foram mais examinadas fisicamente. Esses resultados reforçam a importância da ESF como modelo de organização da atenção, constituindo-se em um modelo mais efetivo, devendo ser ajustado em seus pontos desfavoráveis e ampliado como forma de proporcionar o fortalecimento para reorganização da atenção primaria à saúde.

A subnotificação dos dados registrados pelos profissionais tem sido apontada na literatura como potencial limitação em estudos dessa natureza 2,22,24, assim como o viés recordatório. Para reduzir o possível viés de informação, utilizamos três instrumentos para a coleta dos dados: entrevista, checagem do cartão da gestante e prontuário.

A presente análise não incluiu avaliação de estrutura e resultados, o que permitiria um diagnóstico mais completo da qualidade da atenção pré-natal.

Os resultados deste trabalho poderão contribuir para direcionar a formulação de estratégias que melhorem a qualidade do pré-natal nos dois modelos de atenção, traduzindo-se em uma potente ferramenta para a gestão municipal avaliar a qualidade da assistência pré-natal ofertada à população e o modelo a investir na reorganização da atenção primária à saúde.

Independentemente do modelo de atenção é importante promover a sensibilização e capacitação dos profissionais para melhorar os registros nos cartões das gestantes, implantar e estimular a adesão aos protocolos, realizar avaliações e monitoramentos sistemáticos dos serviços pelos próprios profissionais, e buscar alternativas para promover, junto às gestantes, a continuidade do pré-natal.

A qualidade do pré-natal foi favorável à ESF, contudo, esta ainda tem muito a avançar como modelo de atenção básica, principalmente, quanto à integralidade, longitudinalidade, continuidade e coordenação na consolidação do SUS. 


\section{Resumo}

O processo da atenção pré-natal em unidades básicas de saúde tradicionais (UBS) e unidades de Estratégia Saúde da Família (ESF) foi avaliado em Santa Maria, Rio Grande do Sul, Brasil. Foram entrevistadas 795 puérperas que realizaram pré-natal nas UBS ou ESF. Utilizou-se quatro níveis de qualidade: nível 1 (índice de Kessner modificado por Takeda); nível 2, que adiciona ao nível 1 procedimentos clinico-obstétricos; nível 3, que acrescenta ao nível 1 exames laboratoriais; $e$ nível 4, que considera todos os parâmetros anteriores. A atenção pré-natal realizada na ESF foi superior a das UBS em todos os níveis, com diferenças estatisticamente significativas nos níveis 1 e 2. As gestantes da ESF receberam mais orientações. A atenção pré-natal foi favorável à ESF, devendo ser melhorada em relação aos procedimentos e exames, a fim de aprimorar a assistência pré-natal e fortalecer a atenção primária à saúde.

Cuidado Pré-Natal; Qualidade da Assistência à Saúde; Saúde da Família; Atenção Primária à Saúde

\section{Colaboradores}

E. T. R. Anversa participou da concepção do estudo, da revisão da literatura, coleta dos dados, construção da base de dados, análise dos resultados e redação do texto. G. A. N. Bastos e T. S. Dal Pizzol colaboraram na concepção do estudo, na análise e discussão dos resultados e na revisão do texto. L. N. Nunes contribuiu na análise, discussão dos dados e revisão do texto.

\section{Agradecimentos}

Ao Ministério da Saúde e à Universidade Federal do Rio Grande do Sul pela realização do Mestrado Profissional em Epidemiologia: Gestão de Tecnologias em Saúde. Ênfase em Atenção Primária à Saúde. À Lizandra Flores Pimenta e Dianele Vidal dos Santos, supervisoras de campo. Aos entrevistadores da pesquisa. À direção e aos funcionários do Centro Obstétrico e Maternidade do Hospital Universitário de Santa Maria, e da Maternidade do Hospital Municipal Casa de Saúde. Às puérperas deste estudo.

\section{Referências}

1. Donabedian A. The quality of care. JAMA 1988; 260:1743-8.

2. Koffman MD, Bonadio IC. Avaliação da atenção pré-natal em uma instituição filantrópica da Cidade de São Paulo. Rev Saúde Matern Infant 2005; 5 Suppl 1:S23-32.

3. Halpern R, Barros FC, Victora CG, Tomasi E. Atenção pré-natal em Pelotas, Rio Grande do Sul, Brasil, 1993. Cad Saúde Pública 1998; 14:487-92.

4. Coimbra LC, Silva AAM, Mochel EG, Alves MTSSB, Ribeiro VS, Aragão VMF, et al. Fatores associados à inadequação do uso da assistência pré-natal. Rev Saúde Pública 2003; 37:456-62.

5. Villar J, Ba'aqeel $\mathrm{H}$, Piaggio $\mathrm{G}$, Lumbiganon $\mathrm{P}$, Miguel Belizan J, Farnot U, et al. WHO antenatal care randomised trial for the evaluation of a new model of routine antenatal care. Lancet 2001; 357:1551-64.

6. Carroli G, Villar J, Piaggio G, Khan-Neelofur D, Gülmezoglu M, Mugford M, et al. WHO systematic review of randomised controlled trial of routine antenatal care. Lancet 2001; 357:1565-70.

7. Gonçalves CV, Cesar JA, Mendoza-Sassi RA. Qualidade e equidade na assistência à gestante: um estudo de base populacional no Sul do Brasil. Cad Saúde Pública 2009; 25:2507-16.

8. Neumann N, Tanaka OY, Victora CG, Cesar JA. Qualidade e equidade da atenção ao pré-natal e ao parto em Criciúma, Santa Catarina, Sul do Brasil. Rev Bras Epidemiol 2003; 6:307-18.

9. Trevisan MR, De Lorenzi DRS, Araújo NM, Ésber K. Perfil da assistência pré-natal entre usuárias do Sistema Único de Saúde em Caxias do Sul. Rev Bras Ginecol Obstet 2002; 24:293-9.

10. Rasia ICRB, Albernaz E. Atenção pré-natal na Cidade de Pelotas, Rio Grande do Sul, Brasil. Rev Bras Saúde Matern Infant 2008; 8:401-10.

11. Lansky S, França E, César CC, Monteiro Neto LC, Leal MC. Mortes perinatais e avaliação da assistência ao parto em maternidades do Sistema Único de Saúde em Belo Horizonte, Minas Gerais, Brasil, 1999. Cad Saúde Pública 2006; 22:117-30. 
12. Silva AMR, Almeida MF, Matsuo T, Soares DA. Fatores de risco para nascimentos pré-termo em Londrina, Paraná, Brasil. Cad Saúde Pública 2009; 25:2125-38.

13. Kessner DM, Singer J, Kalk CW, Schlesinger ER. Infant death: an analysis of maternal risk and health care. Washington DC: Institute of Medicine, National Academy of Science; 1973.

14. Kotelchuck M. An evaluation of the Kessner adequacy of prenatal care index and a proposed adequacy of prenatal care utilization index. Am J Public Health 1994; 84:1414-20.

15. Takeda S. Avaliação de unidade de atenção primária, modificação dos indicadores de saúde e qualidade da atenção [Dissertação de Mestrado]. Pelotas: Faculdade de Medicina, Universidade Federal de Pelotas; 1993.

16. Ministério da Saúde. Portaria GM no. 569/2000. Institui o Programa Pré-natal e Nascimento. http:// www.datasus.gov.br/sisprenatal/spn_port9.htm (acessado em 10/Mai/2010).

17. Silva JLP, Cecatti JG, Serruya SJ. A qualidade do pré-natal no Brasil. Rev Bras Ginecol Obstet 2005; 27:103-5.

18. Departamento de Atenção Básica, Secretaria de Atenção à Saúde, Ministério da Saúde. Política Nacional de Atenção Básica. Brasília: Ministério da Saúde; 2006. (Série A. Normas e Manuais Técnicos - Série Pactos pela Saúde, 4).

19. Starfield B. Atenção primária: equilíbrio entre necessidades de saúde, serviços e tecnologias. Brasília: Organização das Nações Unidas para a Educação, a Ciência e a Cultura/Ministério da Saúde; 2002.

20. Brasil. Presidência da República. Objetivos de desenvolvimento do milênio: relatório nacional de acompanhamento. Brasília: Instituto de Pesquisa Econômica Aplicada/Secretaria de Planejamento e Investimentos Estratégicos; 2007.
21. Piccini R, Facchini LA, Tomasi E, Thumé E, Silveira DS, Siqueira FV, et al. Efetividade da atenção pré-natal e de puericultura em unidades básicas de saúde do Sul e do Nordeste do Brasil. Rev Bras Saúde Matern Infant 2007; 7:75-82.

22. Silveira DS, Santos IS, Dias-da-Costa JS. Atenção pré-natal na rede básica: uma avaliação da estrutura e do processo. Cad Saúde Pública 2001; 17:131-9.

23. Departamento de Ações Programáticas Estratégicas, Secretaria de Atenção à Saúde, Ministério da Saúde. Pré-natal e puerpério: atenção qualificada e humanizada: manual técnico. Brasília: Ministério da Saúde; 2006. (Série A. Normas e Manuais Técnicos - Série Direitos Sexuais e Direitos Reprodutivos - Caderno, 5).

24. Coutinho T, Teixeira MTB, Dain S, Sayd JD, Coutinho LM. Adequação do processo de assistência pré-natal entre as usuárias do Sistema Único de Saúde em Juiz de Fora (MG). Rev Bras Ginecol Obstet 2003; 25:717-24.

25. Ceccim RB, Ferla AA. Linha de cuidado: a imagem da mandala na gestão em rede de práticas cuidadoras para uma outra educação dos profissionais de saúde. In: Pinheiro R, Mattos RA, organizadores. Gestão em redes: práticas de avaliação, formação e participação na saúde. Rio de Janeiro: Centro de Estudos e Pesquisa em Saúde Coletiva; Instituto de Medicina Social, Universidade do Estado do Rio de Janeiro; 2006.

26. Fonseca SC, Coutinho ES. Fatores de risco para mortalidade fetal em uma maternidade do Sistema Único de Saúde, Rio de Janeiro, Brasil: estudo caso-controle. Cad Saúde Pública 2010; 26:240-52.

Recebido em 25/Jul/2011

Versão final reapresentada em 15/Nov/2011

Aprovado em 12/Dez/2011 